\title{
Editorial
}

\section{Répétons-le bien: notre Mission est un engagement}

uel plaisir de vous présenter notre huitième numéro! LittéRéalite établit graduellement sa raison d'être et s'enracine peu à peu dans nos communauté et conscience littéraires. Sa mission s'est déjà définie et réalise son point. Avant tout, notre revue vise à servir. Servir, c'est de prime abord le refus de tout campanilisme afin d'ouvrir ses pages aux diverses opinions et à tous les points de vues. Ensuite, en particulier, nous désirons servir les voix littéraires franco-ontariennes et canadienne-françaises. Nous avons déjà consacré deux de nos numéros à la littérature contemporaine de l'Ontario et du Québec. D' ailleurs, en vue de constituer une trilogie d'études canadiennes, nous annonçons un troisième numéro spécial (Automne 1993) dévoué à la créativité acadienne. De même, dans les numéros à sujet ouvert, l'un de nos buts, c'est d'encourager la production critique et littéraire et d'offrir donc aux écrivains ainsi qu'aux critiques de chez nous un moyen de diffuser et de préserver leurs textes. Nous le considérons comme important de faire une contribution, si modeste soit-elle, à l'enrichissement de notre patrimoine culturel. C'est ainsi que nous parviendrons à préserver dans la mesure du possible les fruits de notre esprit créateur. Enfin, il est essentiel de ne pas entraver cette énergie créatrice dans un contexte eccessivement régional qui risque d'atrophier tout élan d'affirmation culturelle. Nous retenons donc indispensable établir des liens de communication entre notre réalité ontarienne et les autres francophonies du Canada et d'ailleurs. Tout développement au-delà d'un état $d^{\prime}$ incubation doit se réaliser dans un espace sans confins, ni contraintes, doit se nourrir largement des vents intellectuels parvenant des quatre coins du monde. C'est à travers un tel dialogue polyphonique que notre identité s'affirmera d'autant plus distincte. Ecartons-nous donc de tout nombrilisme.

Le nombre grandissant de nos contributeurs et lecteurs, la distinction, «Prix de la Meilleure Revue Francophone» (1992) décernée par la ville de Bergerac et la société «Les Amis de la Poésie,» et les nombreuses expressions de gratitude de la part de nos contributeurs, représentent autant d'indices que nous sommes engagés dans la bonne route et que nos efforts sont appréciés.

Nous cherchons encore et toujours à faire mieux. Je suis heureux donc d'annoncer deux nouveaux rédacteurs adjoints: Hédi Bouraoui (Université York) va s'occuper de nos pages de poésie et Louis Bélanger (Université du Nouveau-Brunswick à St-Jean) prendra la responsabilité de nos services de comptes rendus. En particulier, nous tenons à annoncer dans LittéRéalité les nouvelles parutions des maisons d'édition franco-ontariennes et canadienne- françaises. De plus, dans la mesure du possible, 
nous essayerons d'accueillir des comptes rendus de ces nouveaux écrits. Votre participation active à donner forme à cette vision littéraire est encouragée et vivement appréciée.

Sergio Villani

\author{
VILLE DE BERGERAC \\ :5 \\ 13 et $1+$ juin 1992 \\ JOURNEES DE POESIE \\ 将 \\ DIPLOME DE LA SOCIETE CULTURELLE \\ "LES AMIS DE LA POESIE" \\ s \\ Prix de la Meilleure Revue \\ Francophone
}

Décenné par le jury

avec ses compliments à .I. . .

Sergto Hillani

\title{
Pour LittéRéalité
}

BERGER tC, le $1+$ juin 1992

La Présidente.

fur Deflews
Le Président du Jury,

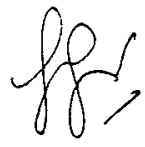

La secrétaire.

Société Culturelle

Les Amis de la Possie

eengerafac?

a. licalors 